\title{
ANÁLISE DO DESEMPENHO DOS ALUNOS DO ITA NO ENADE
}

\author{
Marcos Luciano de Oliveira Santos ${ }^{1}$ \\ Armando Zeferino Milioni ${ }^{2}$
}

Resumo: $\mathrm{O}$ objetivo deste trabalho é executar uma análise estatística sobre desempenho dos alunos do ITA no ENADE. Esta análise inclui os padrōes básicos de comparação entre médias e desvios, ITA versus outras instituições e ITA-2005 versus ITA-2008. O interesse maior está no estabelecimento de métricas comparativas de desempenho dos extremos, com particular interesse no extremo superior.

Palavras-chave: ENADE, avaliação estatística, avaliação ensino engenharia.

Abstract: The main objective in this paper is to perform a statistical analysis on the performance of students from ITA at the ENADE exam. This analysis includes basic comparison of means and standard deviation considering ITA against other institutions and ITA-2005 against ITA-2008. The main interest is in establishing comparative performance metrics on the extremes, with particular interest at the upper end.

Keywords: ENADE, statistical evaluation, assessment engineering education.

\section{INTRODUÇÃO}

\subsection{MOTIVAÇÃO E OBJETIVOS}

Suponha que a elite de uma determinada profissão obtida a partir da conclusão de um curso superior seja composta pelos "N" melhores formandos, a cada ano, no país todo, daquele curso.

Uma questão relevante, então, em diversos aspectos, passa a ser a de se estudar a composição dessa elite.

Por exemplo, em números absolutos, qual a contribuição de cada Instituição de Ensino Superior (IES) nos $\mathrm{N}$ formandos que compõem a elite? $\mathrm{Ou}$, olhando por outro ângulo, qual o percentual de profissionais formados por uma dada IES que pertence à elite?

Tome-se o caso da Medicina, por exemplo.

Dos $\mathrm{N}=100$ (por exemplo) melhores médicos formados no país anualmente, quantos são for- mados na universidade em que você se graduou, na qual você trabalha, na melhor IES pública do seu estado, ou na escola que você tem em mente pela razão que for?

$\mathrm{Ou}$, ainda, de todos os médicos formados nessa mesma escola, quantos fazem parte da elite?

No presente trabalho essa questão é visitada para o caso dos cursos das Engenharia do Grupo III que, de acordo com a portaria 146 do INEP (Portaria INEP, 2009), é composto pelas Engenharias Aeroespacial, Aeronáutica, Automotiva, Industrial Mecânica, Mecânica e Naval.

A IES focada na análise é o Instituto Tecnológico de Aeronáutica (ITA), tradicional escola de engenharia sediada em São José dos Campos e os dados para a análise tomam por base os resultados dos exames ENADE (Exame Nacional de Desempenho dos Estuadantes) realizados em 2005 e 2008.

\footnotetext{
1 marcosluciano@gmail.com, Divisão de Engenharia de Computação, Instituto Tecnológico de Aeronáutica, 12228-900, São José dos Campos, SP

2 milioni@ita.br, Divisão de Engenharia Mecânica-Aeronáutica, Instituto Tecnológico de Aeronáutica, 12228-900, São José dos Campos, SP
} 


\subsection{PRELIMINARES}

O ENADE que faz parte do Sistema de Avaliação de Educação Superior (SINAES), busca aferir o rendimento dos alunos nos cursos de ensino superior de acordo com seus respectivos conteúdos programáticos. O ENADE obtém seus resultados através de amostragem, analisando alunos que ingressam e se formam nas IES de todo o país.

O exame realizado em 2008 contou com a participação de 2.367 IES. Foram analisados 24.842 cursos de graduação do país, englobando 564.415 universitários inscritos, dos quais constam ingressantes e concluintes. Os resultados foram divulgados em meados de 2009.

É sabido que há problemas relacionados ao ENADE. Há casos de cursos inscritos na modalidade errada e também de alunos que boicotam ou desdenham a prova, comparecendo a ela, mas deixando-a em branco, ou fazendo um esforço mínimo de resolve-la.

A abrangência em relação ao número de IES, contudo, e o fato de ser um exame comum em cada modalidade específica contribuem para fazer do ENADE um instrumento de aferição dos desempenhos não apenas dos alunos, como também das IES que os formam.

Assim, pela ausência de alternativas na obtenção de dados e ciente de algumas fragilidades dos resultados fornecidos pelo ENADE, este trabalho adota seus relatórios como fonte de dados da pesquisa.

O trabalho é centrado na análise dos resultados das Engenharias do grupo III e focado na comparação dos resultados obtidos pelo ITA com os resultados obtidos pelas outras IES que formam os mesmos profissionais.

Uma observação de grande relevância no exame realizado em 2008 é a não participação da UNICAMP e da USP, instituições conhecidas pela excelência de seus cursos. Por ausência de dados, portanto, essas duas universidades ficam subtraídas do universo estudado.

A última observação a ser ressaltada é a inclusão do curso de Engenharia Civil do ITA dentro do Grupo III, mesmo existindo uma prova específica para essa modalidade de Engenharia. Em 2005 este curso foi corretamente cadastrado pelo ITA. Entretanto, por razões desconhecidas, em 2008 houve esse equívoco de cadastramento. Assim, os resultados do ENADE para o ITA referem-se aos cursos de Engenharia MecânicaAeronáutica, Aeronáutica e Civil.

Infelizmente os resultados divulgados pelo INEP não permitem a desagregação por modalidade. Assim, há que se reconhecer que as análises feitas, conquanto continuem possíveis, ficam prejudicadas por esse equívoco cadastral. Sua gravidade, todavia, é relativamente atenuada pelo fato de que dos 62 alunos do ITA que fizeram o exame apenas 12 (menos de 20\%) eram alunos da Engenharia Civil.

\section{RESULTADOS DO ENADE}

\subsection{DADOS GERAIS PARA AS ENGENHARIAS DO GRUPO III}

Em 2008 foram 4.429 os formandos no Brasil, Deles, $72 \%$ realizaram o exame do ENADE. O ITA teve 62 formandos, dos quais 100\% realizaram o exame. As IES que serviram como base de comparação com o ITA por estarem entre as nove melhores médias do Brasil foram (em ordem decrescente das médias institucionais): IME, UFSC, UTFP, UNIFEI, UNESP, UFU, UFES e UNIOESTE.

Abaixo segue a Tabela com os principais dados extraídos dos relatórios dessas IES. Temse também o resultado geral do Brasil, para que possa também ser comparado. Todos os resultados foram retirados dos Boletins de Desempenho do ENADE (ENADE, Boletins de Desempenho, 2009).

Na Tabela 1, Pop significa População Total, ou número de formandos da IES, Pres refere-se ao número de alunos presentes ao exame, Méd é a média obtida pelos alunos daquela IES, EPM é o erro padrão da média, DP é o desvio padrão, Med é a mediana e Min e Max são, respectivamente, a menor e a maior nota obtida pelos alunos da IES.

Da Tabela 1, nota-se que $100 \%$ dos alunos formados pelo ITA participaram do ENADE, algo que só aconteceu também com a UNIOESTE. 
Tabela 1 - Dados das Nove Melhores Instituições das Engenharias III

\begin{tabular}{|c|c|c|c|c|c|c|c|c|c|c|}
\hline & BRASIL & ITA & IME & UFSC & UTFPR & UNIFEI & UNESP & UFU & UFES & UNIOESTE \\
\hline Pop & 4429 & 62 & 9 & 83 & 44 & 74 & 59 & 80 & 74 & 16 \\
\hline Pres & 3174 & 62 & 7 & 45 & 35 & 46 & 43 & 55 & 52 & 16 \\
\hline Méd & 45,1 & 62,6 & 67,8 & 60,9 & 57,0 & 55,6 & 54,5 & 54,0 & 52,8 & 52,0 \\
\hline EPM & 0,2 & 1,4 & 3,0 & 1,3 & 1,4 & 1,3 & 1,1 & 1,3 & 1,4 & 3,3 \\
\hline DP & 12,9 & 10,7 & 9,0 & 12,1 & 9,0 & 11,5 & 8,6 & 11,5 & 12,2 & 13,3 \\
\hline Med & 44,9 & 63,7 & 64,6 & 62,9 & 56,5 & 56,4 & 54,6 & 57,1 & 53,2 & 54,7 \\
\hline Min & 0,0 & 33,9 & 57,4 & 12,8 & 39,3 & 25,5 & 33,9 & 27,2 & 19,1 & 23,8 \\
\hline Max & 87,6 & 85,6 & 82,4 & 79,3 & 75,0 & 79,1 & 73,5 & 76,5 & 77,8 & 70,4 \\
\hline
\end{tabular}

A média geral mostra o IME em primeiro lugar, $8,3 \%$ acima da média do ITA. A UFSC teve um resultado próximo ao do ITA, estando o ITA $2,7 \%$ a sua frente. As demais IES têm médias mais do que $10 \%$ abaixo da média do ITA.

A Figura 1 exibe uma perspectiva geral do desempenho das IES.

Figura 1 - Notas por Instituição

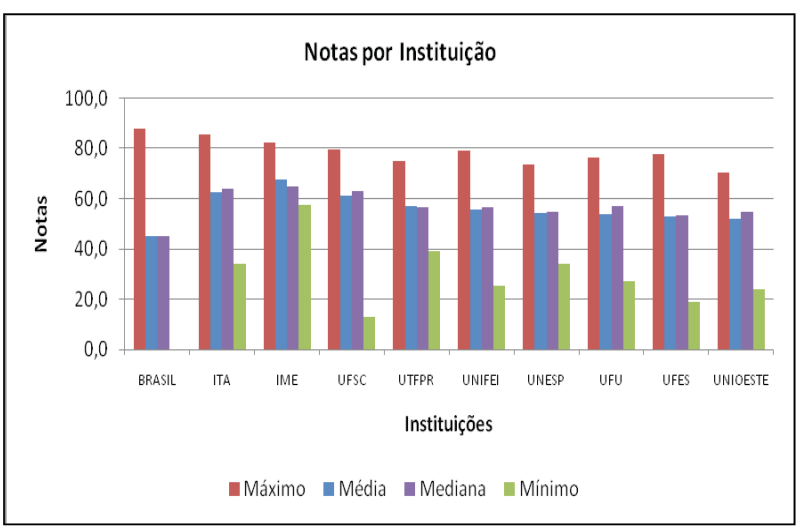

Os valores das medianas mostram novamente o IME melhor classificado, 1,4\% acima do ITA, segundo melhor classificado. A UFSC teve um resultado próximo ao do ITA, com a diferença de $1,3 \%$. As demais IES têm mediana mais do que $10 \%$ abaixo da mediana do ITA.

Cabe ressaltar também que em todas as colunas da Tabela os valores das médias e das medianas são bastante próximos (a diferença média é inferior a 3\%).

O desvio-padrão de cada instituição dá uma referência de quão dispersos são os rendimentos dos alunos. A UNESP aparece como a instituição detentora do menor desvio, cerca de 19,6\% me- nor do que o do ITA, que ficou em quarto lugar nessa classificação.

A nota máxima nacional não foi atingida por nenhuma das nove IES envolvidas nos estudos. A nota do melhor aluno do Brasil ficou 2\% acima da nota do aluno que atingiu a maior nota dentre os alunos do ITA. As demais instituições de ensino obtiveram resultados variando de 3,7\% a 17,8\% inferiores à máxima do ITA.

Quanto à nota mínima dos alunos de cada IES, há três IES com notas mínimas maiores do que a obtida pelo ITA. Nesse quesito, o IME, que é o primeiro colocado, obteve uma nota superior ao ITA em $69,3 \%$.

\subsection{DESEMPENHO POR COLOCAÇÃO}

As análises que seguem procuram identificar quantos alunos de cada IES aparecem nas faixas dos primeiros $\mathrm{N}$ colocados para diferentes valores de $\mathrm{N}$.

Os resultados foram obtidos a partir da hipótese de que as notas de cada IES seguem uma distribuição Normal com média e desvio dados pelos valores apresentados na Tabela 1 .

A primeira linha da Tabela 2 (Colocações) traz os diferentes valores de N. A segunda linha (\%Sup) traz o \% superior a que se refere N. Por exemplo, se $\mathrm{N}=100$, então, considerando que 3.174 alunos fizeram o exame, os 100 primeiros correspondem aos 3,2\% (resultado aproximado de 100 x 100 / 3.174) superior da distribuição. A $3^{a}$ linha (Nota) traz a estimativa da nota (de 0 a 100) necessária para que o aluno esteja colocado entre os $\mathrm{N}$ primeiros. Após essas três primeiras linhas, seguem-se duas linhas para cada IES. 
Tabela 2 - Distribuição dos alunos de cada IES por faixa de colocação

\begin{tabular}{|c|c|c|c|c|c|}
\hline Colocaçōes & 5 & 10 & 50 & 100 & 200 \\
\hline$\%$ Sup & $0,16 \%$ & $0,32 \%$ & $1,6 \%$ & $3,2 \%$ & $6,3 \%$ \\
\hline Nota & 83,2 & 80,3 & 72,8 & 69,1 & 64,8 \\
\hline \multirow{2}{*}{ ITA } & 1 & 3 & 10 & 16 & 25 \\
\hline & $2 \%$ & $5 \%$ & $16 \%$ & $26 \%$ & $40 \%$ \\
\hline \multirow{2}{*}{ IME } & 0 & 0 & 2 & 3 & 4 \\
\hline & $0 \%$ & $0 \%$ & $29 \%$ & $43 \%$ & $57 \%$ \\
\hline \multirow{2}{*}{ UFSC } & 1 & 2 & 7 & 11 & 16 \\
\hline & $2 \%$ & $4 \%$ & $16 \%$ & $24 \%$ & $36 \%$ \\
\hline \multirow{2}{*}{ UTFPR } & 0 & 0 & 1 & 3 & 6 \\
\hline & $0 \%$ & $0 \%$ & $2 \%$ & $7 \%$ & $14 \%$ \\
\hline \multirow{2}{*}{ UNIFEI } & 0 & 0 & 3 & 5 & 9 \\
\hline & $0 \%$ & $0 \%$ & $7 \%$ & $11 \%$ & $20 \%$ \\
\hline \multirow{2}{*}{ UNESP } & 0 & 0 & 0 & 1 & 4 \\
\hline & $0 \%$ & $0 \%$ & $0 \%$ & $2 \%$ & $9 \%$ \\
\hline \multirow{2}{*}{ UFU } & 0 & 0 & 2 & 5 & 9 \\
\hline & $0 \%$ & $0 \%$ & $4 \%$ & $9 \%$ & $16 \%$ \\
\hline \multirow{2}{*}{ UFES } & 0 & 0 & 2 & 4 & 8 \\
\hline & $0 \%$ & $0 \%$ & $4 \%$ & $8 \%$ & $15 \%$ \\
\hline \multirow{2}{*}{ UNIOESTE } & 0 & 0 & 0 & 1 & 2 \\
\hline & $0 \%$ & $0 \%$ & $0 \%$ & $6 \%$ & $13 \%$ \\
\hline
\end{tabular}

A primeira apresenta a estimativa de quantos alunos daquela IES estão entre os melhores $\mathrm{N}$ colocados. Por exemplo, a estimativa feita aponta para a presença de 16 alunos do ITA entre os 100 primeiros colocados. A segunda linha refere-se a qual o $\%$ de alunos daquela IES que se encontra entre os primeiros $\mathrm{N}$ colocados. Mantendo o exemplo, $26 \%$ dos alunos do ITA (16 em 62) encontram-se entre os primeiros 100 colocados.

Os cálculos foram feitos tomando por base os procedimentos descritos em (BARBETTA e BORNIA, 2009), (DEVORE, 2006) ou (LEVIN, 1987).

Da Tabela 2 observa-se que o ITA é a IES com maior número de alunos (16) dentre os 100 primeiros colocados no exame (faixa considerada a elite da modalidade). Esses 16 alunos representam $26 \%$ dos 62 alunos do ITA que fizeram o exame.

Em termos percentuais, os $26 \%$ do ITA ficam aquém dos 43\% do IME (embora os números pequenos prejudiquem a análise) e muito próximos dos 24\% (ou 11 alunos em 45) da UFSC.

Mantendo o foco no ITA, $66 \%$ de seus alunos (41 em 62) aparecem entre os primeiros 500 colocados do exame. Isso significa que há 21 alunos além da posição de número 500 .

Esse número surpreende, mesmo considerando que entre esses 21 alunos podem estar os 12 alunos de Engenharia Civil inadvertidamente incluída no grupo das Engenharias III.

As figuras 2 e 3 , que seguem, trazem as distribuições normais assumidas para cada IES analisada.

$\mathrm{Na}$ figura 2 as distribuiçôes aparecem em tamanho absoluto, o que significa dizer que a área sob a curva corresponde ao total de alunos de cada IES.

Figura 2 - Distribuição dos alunos, em números absolutos

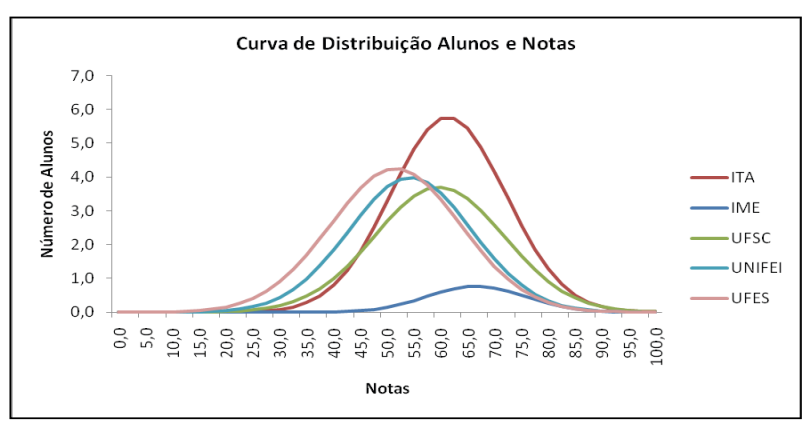


$\mathrm{Na}$ figura 3 as distribuiçôes aparecem com a mesma média e desvio, o que significa que a integral sob todas elas é comum e igual a $100 \%$.

Com as figuras 2 e 3 percebe-se nitidamente o desempenho semelhante dos alunos da UFSC e do ITA. Também é visível o destaque do IME (figura 3), apesar do baixo número de alunos da sua amostra (figura 2).

Figura 3 - Distribuição dos alunos, em freqüência relativa

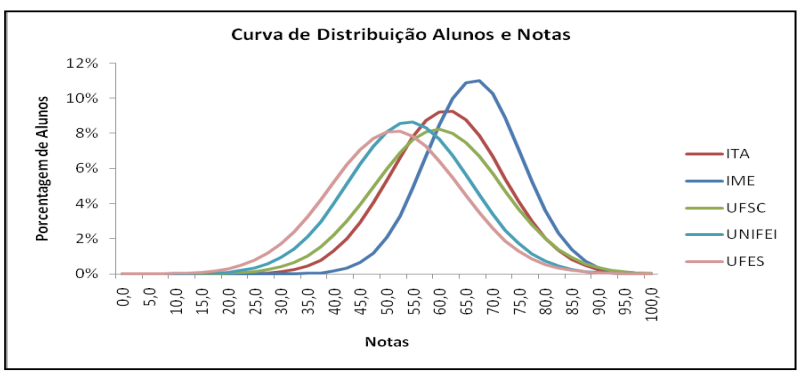

\subsection{TESTE DE COMPARAÇÃO DAS MÉDIAS}

Para aprofundamento dos resultados até então apresentados realizou-se o Teste $T$ de igualdade entre a média de cada IES comparada à média do ITA sob a hipótese de desvio padrão comum para todas as IES. Esse teste é descrito, por exemplo, em (DEVORE, 2006). Ele demanda a estimação desse desvio padrão comum (pooled standard deviation) obtida pela ponderação das variâncias em função do tamanho da amostra em cada IES. Detalhes podem ser encontrados em (DE MARTINS e DONAIRE, 2006) e (DE MARTINS, 2009).

Os resultados são apresentados na Tabela 3.

Tabela 3 - Resultados do Teste T

\begin{tabular}{|c|c|c|c|c|c|c|c|}
\hline & IME & UFSC & UTFPR & UNIFEI & UNESP & UFU & UFES \\
\hline DifM & $-5,2$ & 1,7 & 5,6 & 7,0 & 8,1 & 8,6 & 9,8 \\
\hline NívSig & $11,1 \%$ & $22,7 \%$ & $0,5 \%$ & $0,1 \%$ & $0,0 \%$ & $0,0 \%$ & $0,0 \%$ \\
\hline
\end{tabular}

Na primeira linha dessa Tabela, DifM traz as diferenças entre a médias obtida pela respectiva IES e a média do ITA, igual a 62,6 (conforme Tabela 1). Na segunda linha, identificada por NivSig (referente a Nível de Significância) encontram-se valores $\mathrm{p}$ dos testes a partir dos quais é possível rejeitar a hipótese nula de igualdade entre as médias do ITA e da IES analisada na respectiva coluna

Note-se que só é possível afirmar que a média do IME é diferente da do ITA a partir de um nível de significância igual a $11,1 \%$, que praticamente nunca é usado em análises estatísticas convencionais. De fato, se for adotado o nível de significância padrão de 5\%, os resultados apresentados na Tabela 3 mostram que a média dos alunos do ITA não é diferente das obtidas pelos alunos do IME e da UFSC, sendo, todavia, nitidamente diferente - e superior - às médias obtidas pelos alunos de todas as outras IES analisadas.

\section{ITA: COMPARAÇÃO DOS RESULTADOS 2008 E 2005}

É analisado, nesta seção, o desempenho dos formandos do ITA de 2008, em relação aos for- mandos de 2005. Para outra referência além do ITA, foram feitas observações a respeito do desempenho nacional, assim, de certa forma, procurou-se balizar os efeitos de dois exames distintos realizados em anos distintos.

Uma diferença existente entre 2005 e 2008 é a colocação do curso de Engenharia Civil na modalidade Grupo III. Em 2005 este curso foi avaliado em sua modalidade, e como já observado em 2008, teve sua classificação alterada. Com isso, avalia-se e compara-se a modalidade Grupo III de 2005, onde estavam inclusos os cursos de Engenharia Mecânica-Aeronáutica e Engenharia Aeronáutica, com o de 2008, onde se encontra adicionado ainda o curso Engenharia Civil.

Nos estudos iniciais compara-se o desempenho nacional de 2008 em relação ao ano de 2005. O que se percebe é uma leve diminuição no resultado das notas. Os relatórios dos exames em 2008 mostram um aumento em $23 \%$ da população de formandos nesta modalidade e um aumento de $43 \%$ no grupo de formandos que foram avaliados, isto é, o ENADE 2008 conseguiu mais abrangência em relação ao exame de 2005. 
Do resultado das notas nacionais tem-se, em 2008, uma média $8 \%$ inferior e uma mediana $9 \%$ também inferior.

Este resultado não implica, necessariamente, queda no nível dos alunos, dado que as provas dos exames são diferentes. Outro fato importante a ser avaliado é a queda no valor do desvio-padrão, de $15 \%$, isto reflete uma maior coesão no resultado dos exames dos formandos, ressaltandose que esta redução no desvio se deu perante a um aumento no espaço amostral avaliado. A Figura 4 mostra estas relações.

\section{Figura 4 - Comparação Brasil - Engenharia Grupo III 2008 x 2005}

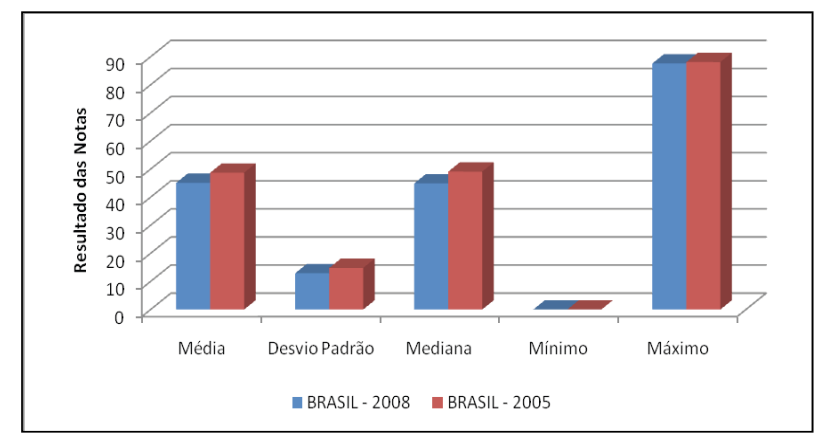

Nos resultados do ITA, encontram-se, também em 2008, números não superiores a 2005 . O conjunto de formandos que realizou a prova foi $27 \%$ maior e o motivo disso é a inclusão da Engenharia Civil nesta modalidade. $\mathrm{O}$ número de formados no ITA tem se mantido quase invariante no tempo.

\section{Figura 5 - Comparação ITA - Engenharia Grupo III 2008 x 2005}

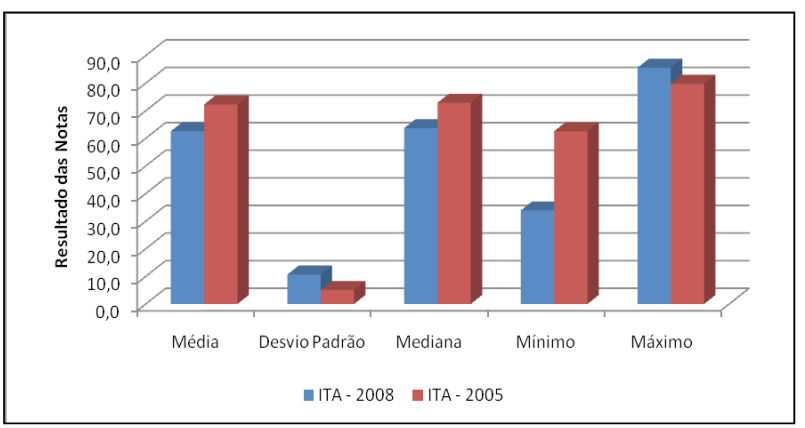

Do resultado das notas, na modalidade Grupo III do ITA, tem-se uma média 16\% inferior e uma mediana $14 \%$ também inferior. Este resultado também não expressa claramente uma queda no nível dos alunos, dado que as provas dos exames são diferentes e o resultado nacional tam- bém teve uma baixa. Outro dado importante a ser avaliado é o aumento no valor do desvio-padrão de $52 \%$ que reflete a maior dispersão no resultado dos exames dos formandos. Mais uma vez, é importante salientar que a presença dos formandos de Engenharia Civil nesta modalidade à qual não pertence gera conseqüências negativas nestes resultados. A Figura 5 mostra todas estas relações.

\section{COMENTÁRIOS}

O ITA é uma instituição que projeta e goza de uma imagem de excelência. Os alunos aprovados no vestibular recebem bolsa integral que inclui alimentação e moradia no campus por um valor de aluguel praticamente simbólico. Eles assumem ainda o compromisso de dedicação exclusiva ao curso que é ministrado em período integral. Por todas essas razões, os aluno e ex-alunos do ITA costumam repetir que um mau aluno do ITA ainda é melhor do que bons alunos de outras escolas de engenharia. Essa afirmação não parece sustentável à luz das seguintes evidências: (i) a média dos alunos do ITA nas Engenharias do Grupo III não é estatisticamente diferente da de seus pares de pelo uma outra IES, no caso, a UFSC. O desvio padrão das notas dos alunos do ITA é superior ao de 3 outras IES analisadas, indicando maior dispersão.

Também surpreende que apenas 16 dos 62 alunos consigam desempenho suficiente para se posicionar na "elite" dos 100 melhores formandos de 2008. Juntas, as outras 8 IES estudadas posicionam o dobro (33) alunos nessa mesma elite, o que significa que metade dessa elite é composta de alunos do ITA e das outras 8 IES analisadas.

Finalizando, cumpre lembrar que se, por um lado, no caso do ITA, os alunos da Engenharia Civil foram inadvertidamente incluídos na análise, também é verdade, por outro lado, e, de certa forma, compensatório, que escolas tradicionais como Unicamp e USP não foram incluídas nessa mesma análise.

\section{REFERÊNCIAS}

LEVIN, Jack, Estatística Aplicada a Ciências Sociais, Harbra ltda, 1987.

DE MARTINS, G. A. e DONAIRE, Denis, Princípios de Estatística, Atlas, 2006. 
DE MARTINS, G. A., Estatística Geral e Aplicada, Atlas, 2009.

BARBETTA, Alberto, REIS, M. M. e BORNIA, A. C., Estatístca: para Cursos de Engenharia e Informática, Atlas, 2009.

DEVORE, Jay L., Probabilidade e Estatística para Engenharia e Ciências, Pioneira Thomson, 2006.
PORTARIA INEP NO 146, Seção 1, pág.32. Disponível em: <http://www.mecanica.ufrgs.br/ comgrad/Diretrizes_Engenharias_n_146.pdf> Acesso em: 22 set. 2009.

RESULTADOS ENADE, Boletim de Desempenho, Disponível em: <http://enade.inep. gov.br/enadeResultado/site/recuperarSenha. seam?cid=41288>. Acesso em: 05 out. 2009. 